\title{
The interplay between chemical reactions and transport in structured spaces
}

\author{
Zoran Konkol:* \\ Department of Applied Physics, \\ Chalmers University of Technology and Göteborg University, \\ SE-412 96 Göteborg, Sweden
}

(Dated: August 18, 2018)

\begin{abstract}
There are many instances in nature where geometry of the system that sustains chemical reactions is structured and the living cell is a typical example. There is a high degree of compartmentalization in the living cell where various compartments sustain different chemical reactions and transport reactants among themselves. In order to avoid storage facilities reactants are routed in orderly fashion between various places in the cell interior with large degree of synchronization. It is exactly such situation that is investigated here. The main motivation behind this study is to understand interplay between reactions and transport in a geometries that are not compact. Typical examples of compact geometries are box, sphere, etc. On the other hand, a network made of containers $C_{1}, C_{2}, \ldots, C_{N}$ and tubes is a typical example of space that is structured, and such non compact space is main focus on this study. The whole space is divided into a two regions. First, in containers particles react with rate $\lambda$. Second, tubes connecting containers allow for exchange of chemicals with transport rate $D$. In such a way the two most important processes are isolated in the problem, reactions and transport. By varying topology of such network and details of chemical reactions it is possible to gain some understanding of interplay between chemical reactions and transport in structured spaces. It assumed that the number of reactants in the system is so small that kinetics is noise dominated. Two methods for solving corresponding master equation are discussed. The computer simulation is easy to implement, but leads to the results that are not that accurate. In here, a method is presented that can be used to calculate average, variance, and higher moments of the time reaction needs to finish. The method relies on a matrix representation of the master equation and is in principle exact. It works for an arbitrary reaction scheme and network topology. A number of different chemical reactions were studied and their performance compared in a various ways. Reactions are grouped into two ensembles, Reaction on a Fixed Geometry Ensemble (ROGE) and Geometry on a Fixed Reaction Ensemble (GORE). The ROGE and GORE are used to classify reactions in order to gain some understanding of which types of chemical reactions draw most benefit from the structured spaces. Most important findings are as follows. (i) There is a large number of reactions that run faster in a network like geometry. Such reactions contain antagonistic catalytic influences in the intermediate stages of a reaction scheme that is best dealt with in a network like structure. (ii) Antagonistic catalytic influences are hard to identify since they are strongly connected to the pattern of injected molecules (inject pattern) and depend on the choice of molecules that have to be synthesized at the end (task pattern). (iii) The reaction time depends strongly on the details of the inject and task patterns.
\end{abstract}

\section{INTRODUCTION}

The goal of the present study is to impact some progress in understanding interplay between reactions and transport in structured spaces. There are many questions one could ask. This study focuses on one: How strongly the geometry of the system (e.g., topology and shape of the boundaries) influences the particular reaction schemes it harbors? This question can be asked in two ways. First, assuming that spatial structure is fixed, which reaction scheme would draw most benefit from it, for example, in terms of speed (smaller execution time) or better timing (reduction of noise level)? Second, given the chemical reaction, what is the topology that is mostly suited for it? In order words, in which ways should one alter geometry of the system (without

*Electronic address: zorank@fy.chalmers.se altering reactants or influencing reaction mechanisms in any other way) in order to speed up chemical reaction encapsulated inside? Answers to these questions are relevant to a number of topics ranging from understanding of physio-chemical processes in the living cell, biological evolution, towards chemical engineering and biotechnology.

Taking the living cell as an example. The living cell exhibits large degree of spatial organization. Various compartments sustain different chemical reactions and transport reactants among themselves, with a high degree of spatial and temporal synchronization. Reactants and products of reaction are transported where they are needed, exactly at the right place and at the right time. There is no need of excessive storage of molecules. For reviews on these topic see 1, 2], and refs. 3, 4, 5, 6, 7, 8, 9, 10, 11, 12] for other interesting work. The topics related to temporal and spatial synchronization of the cellular machinery are still hotly debated and present work might offer some understanding 
of these phenomena. Furthermore, thinking from evolutionary perspective, one wonders what is that cells try to optimize by adopting highly structured geometry?

Evolutionary process occurred in two stages: the chemical evolution [13], which lead to emergence of organic molecules necessary for synthesis of proteins and anything alive, followed by biological evolution 14], a process which started after emergence of the first living cell. Fairly little has been done in understanding the role of geometry and spatial organization played in the evolution process. There is already some pioneering work in this area 15, 16, 17, 18, 19]. Present study could impact some progress in this direction.

Besides the topics discussed, the question of interplay between geometry and reaction scheme is relevant to a number of disciplines. The chemical engineering and biotechnology are typical examples. In general, there is a tendency to move away from bulk situation where volumes are large and reactants many, towards more exotic regime where reaction volumes are small and structured and number of reactants relatively few. The interesting experimental work on these topics can be found in refs. 20, 21, 22, 23] and 24, 25, 26]. The first set of references deals with reactions in liposome networks, and second set addresses the situation where a number of perfectly stirred reactors is connected by tubes. The exchange of chemicals through tubes is driven by pumps. The work done in here is relevant to both of these studies.

The particular model proposed here is influenced by several lines of research. The setup of the reaction schemes is inspired by the work done on prebiotic evolution, genome-based evolution and random reaction networks 27, 28, 29, 30, 31, 32, 33, 34), and diffusioncontrolled reactions in bulk phase 35, 36, 37, 38, 39, 40 and in restricted geometries 41, 42, 43, 44. The geometrical setup of the model is motivated by the experimental studies given in 20, 21, 22, 23, 24, 25, 26] and theoretical study of reaction-diffusion neuron and enzymatic neuron 45, 46, 47, 48, 49, 50, 51.

The situation considered here is radically different from the bulk studies. The goal is to mimic particular aspect of the cell environment where reactions happen in the specific regions of space and reactants move among these regions. The simplest way of achieving such a setup is to consider chemical reactions in containers that are connected by tubes. Figure 1 shows one possible example. Reactants can move from container to another along tubes. Thus, the compartmentalization is build into the model in the simplest possible way. Furthermore, it is assumed that containers are small in size so that the number of reactants in each container is also small. Such setup inevitably leads to fluctuations, and chemical kinetics becomes noisy, as is usual the case in the living cell.

For simplicity reasons, the details, both of the chemical reactions and transport, are neglected to a large extent.
It is assumed that reactants are point like objects with no structure. What is studied here can be classified as artificial chemistry [52]. The two most important time scales are traced in the model. The reaction rate $\lambda$ describes how fast molecules react in containers. The transport rate $D$ governs exchange of chemicals among containers. It is assumed that diffusion is the dominant mechanism of particle transport and this is reasonably good approximation for the living cell. [1, 2] Naturally, there are exceptions to this rule, e.g. transport of molecules by kinesin along microtubule in the cell, but such cases are not going to be considered here. For discussion of reaction-diffusion systems in biology please see ref. 53.

How to compare chemical reactions in a compact and structure geometries depicted in Fig. 1 [panels (a) and (b)]? Naturally, the most obvious way would be to run chemical dynamics directly on structures depicted in panels (a) and (b) and try to trace differences. However, it will be shown that this can also be done by studying kinetics in network like geometry depicted in panel (c), but such strategy has to be implemented carefully. For example, it is easy to see that panel (c) in Fig. प1 is a very rough representation of structured geometry in panel (b). However, there is no apparent similarity between structures shown in panels (a) and (c). Nevertheless, the network structure in (c) contains (a) and (b) as special cases. For example, when $D \sim \lambda$ the reaction dynamics occurring in the network depicted in panel (c) should exhibit roughly the same features as the reaction dynamics running in the structured geometry depicted in panel (b). Furthermore, when transport rate dominates all other processes in the system, $D \gg \lambda$, same should hold for (a) and (c). Also, in such a case, $D$ should be much larger than any other reaction rate that might enter due to the effects of catalysis. Thus, in order to gain some understanding of differences between (a) and (b) one simply has to study variations of a reaction dynamics in the network like structure (c) as transport rate changes from $D \sim \lambda$ towards $D \gg \lambda$.

The type of the analysis used is largely static. To understand in which ways particular shape of the reaction volume influences chemical kinetics (and vise versa) a large number of chemical reactions will be analyzed and their performance compared. The set of reactions and geometries chosen form an ensemble $\mathcal{A}=\left\{o_{\omega}, \omega=\right.$ $1, \ldots, E\}$ where elements in ensemble are pairs $o_{\omega}=$ (reaction, geometry). The element $o_{\omega}$ will be referred to as an organism.

Two ensembles are distinguished. In the first case, the reaction scheme is kept fixed, while the geometry of the network is allowed to change. This type of ensemble will be referred to as the geometry on the (fixed) reaction ensemble (GORE). The geometries are different and they are sampled by changing size of the containers and length of the tubes. In the second case the geometry of the network is kept fixed, while the reaction scheme is subject to a change. This type of the ensemble will be referred to as 
the reaction on the (fixed) geometry ensemble (ROGE). In this work most of the attention is on the ROGE ensemble.

What is the measure of the good performance for a chemical reaction? In here, focus will be on the time related issues such as the length of catalytic cycles. The importance of the timing in the intra cellular process has been discussed by Volkenstein [14]: 'The most important role in ontogeny is played by time factors, i.e. the time relations in the synthesis of various proteins and the formation of various cells and tissues. Even small changes in the timetable lead to considerable morphological alternations...'. The setup presented here allows for consideration of other performance criteria but these are omitted due to the simplicity reasons.

The time needed for a reaction to finish, $\mathcal{T}$, is a stochastic variable and for a given reaction scheme and topology it will have well defined distribution function $\Phi(t)$ : the probability that reaction finished within the time interval $[t, t+d t]$ is given by $P(t<\mathcal{T}<t+d t)=$ $\Phi(t) d t$. All information about reaction timing is hidden in the distribution function $\Phi(t)$. In practise, it is very hard to find $\Phi(t)$ for general reaction scheme and geometry. Also, amount of information contained in $\Phi(t)$ is too large. It is more fruitful to characterize $\Phi(t)$ in terms of few variables and in this work we use two: the average time needed for reaction to complete $\tau=\int_{0}^{\infty} t \Phi(t) d t$ and the variance $\sigma$ where $\sigma^{2}=\int_{0}^{\infty}(t-\tau)^{2} \Phi(t) d t$. In such a way it is possible to asses how fast reaction happens and how noisy it is.

Once performance criteria are quantified one faces optimization problem of finding the best performer in a given ensemble $\mathcal{A}$. In principle, the best performer will be the organism that exhibits smallest value for $\tau$. Two strategies are used to find such organism. First, one simply generates full ensemble, measures performance of each element, and sorts performance at the end. However, when number of elements in such ensemble is large another optimization method has to be used. For example, when there is a need for that, one can use a method described in refs. [45, 46, 47, 48, 49, 50, 51]. This optimization method mimics process of evolution and belongs to the class of genetic optimization algorithms. The terms evolution and optimization will be used interchangeably through out the text.

The paper is organized as follows. In section [1] the reaction-diffusion model is defined in detail, and in section [III overview of the methods for solving the master equation is given. The method of doing a computer simulation is reviewed first. The method of finding moments of the time length of the catalytic cycles is discussed in detail in the next section IV Few simple reactions are discussed in section $\nabla$ where it is shown how to compare vastly different reaction schemes. Section VI contains analysis of ROGE ensemble made from two container network, two particle types, all possible reactions, and full set of inject-task patterns. Conclusions and outlook are given in section [DII

\section{THE MODEL}

The reaction-diffusion model is defined as follows. Consider the set of containers $C_{i}$ connected in a particular way by tubes with lengths $l_{i, j} ; i, j=1, \ldots, N$. It is possible that some containers are not connected. Example of such structure is given in Fig. 1. The containers harbor molecules $X_{\alpha} ; \alpha=1,2, \ldots, M$. Molecules are allowed to react only when in the same container (provided there is a reaction they participate in). Molecule $X_{\alpha}$ moves from the container $C_{i}$ to the container $C_{j}$ with a rate $D_{i j}^{\alpha \alpha}$. Please note that the expression for the transport rate is diagonal in the particle index $\alpha$ (there is no change of particles while being transported). This assumption could be easily relaxed. For simplicity reasons it is assumed that $D_{i j}^{\alpha \alpha}=f\left(l_{i j}\right)$ if the link between container $i$ and $j$ exists, otherwise $D_{i j}^{\alpha \alpha}=0$. Links are nondirectional and transport rates are same for all particles, i.e. $D_{i j}^{\alpha \alpha}=D_{i j}=D_{j i}$. Please note that the regular lattice is a special case of this very general model. Structures like these can be found in the interior of the living cells. Mapping is not exact, but there is a rough similarity.

By assumption, the reactions only occur in the containers and not in the tubes, and reactants mix well in the containers. Tubes serve only as connectors of regions where reactions happen. With assumptions at hand, to describe system at any time instant, it is sufficient to track number of particles in each container. This greatly simplifies calculations. Conformation of the system $\boldsymbol{c}$ is specified as a occupancy of the containers,

$$
\boldsymbol{c}=\left(\boldsymbol{n}_{1}, \ldots, \boldsymbol{n}_{i}, \ldots, \boldsymbol{n}_{N}\right)
$$

where vectors $\boldsymbol{n}_{i} i=1, \ldots, N$ describe particle content of each container,

$$
\boldsymbol{n}_{i}=\left(n_{1, i}, \ldots, n_{\alpha, i}, \ldots, n_{M, i}\right)
$$

with $n_{\alpha, i}=0,1,2, \ldots, \infty$ for $\alpha=1, \ldots, M$ and $i=1, \ldots, N$. System makes random transitions between various configurations. A configuration of the system, $\boldsymbol{c}$, changes when either reaction or transport occur.

For computational convenience, the very simple type of a reaction scheme will be considered,

$$
X_{\alpha} \stackrel{ \pm X_{\gamma}}{\rightarrow} X_{\beta}
$$

where $X_{\gamma}$ is understood to influence conversion of $X_{\alpha}$ to $X_{\beta}$ either as catalyst (+) or suppressor (-). All reactions that are allowed are assumed to have same reaction rate $\lambda$. The reaction graph is specified by the reactivity matrix $\boldsymbol{\Lambda}$. If reaction $X_{\alpha} \rightarrow X_{\beta}$ is allowed $\Lambda_{\alpha, \beta}=1$ and 
equals zero otherwise. The reaction graph is directed and matrix $\boldsymbol{\Lambda}$ is not necessarily symmetric.

The effect of catalyst or suppressor are modeled as follows. The bare reaction rate $\lambda$ may be modified by the presence of other reactants in the container $C_{i}$. It is assumed that each reaction can have at most one catalyst or suppressor. On the other hand, it is possible that one particle is catalyst (or suppressor) for more than one reaction. For programming purposes it is sufficient to use array $K_{\alpha, \beta}= \pm \gamma$ if $X_{\gamma}$ is catalyst $(+)$ or suppressor (-) for $X_{\alpha} \rightarrow X_{\beta}$ reaction. $K_{\alpha, \beta}=0$ indicates that reaction does not have catalyst nor suppressor. Also, it is assumed that the effects of the catalysis are strongly enhanced. If there is some catalysis going on, it completely dominates reaction scheme. Using these assumptions the reaction rate for the reaction $X_{\alpha} \rightarrow X_{\beta}$ in the container $C_{i}$ is given by

$$
\lambda_{\alpha, \beta}^{i}\left(\boldsymbol{n}_{i}\right)=\lambda \Lambda_{\alpha, \beta} \begin{cases}1 & \kappa=0 \\ \xi & \kappa>0, n_{\kappa, i}>\delta_{\kappa, \alpha} \\ \frac{1}{\xi} & \kappa<0, n_{|\kappa|, i}>\delta_{|\kappa|, \alpha}\end{cases}
$$

$\delta_{\alpha, \beta}$ is a Kronecker delta-function, $\xi>1$ denotes catalysis enhancement factor, and $\kappa=K_{\alpha, \beta}$. Equation (4) is self explanatory for the exception of possibly one term that we proceed to discuss. The condition $n_{\kappa, i}>\delta_{\kappa, \alpha}$ ensures that there is no self catalysis for the reaction of the type

$$
X_{\alpha} \stackrel{X_{\alpha}}{\rightarrow} X_{\beta}
$$

when there is only one $X_{\alpha}$ present in the container. One needs at least two $X_{\alpha}$ in order to fell catalytic influence of $X_{\alpha}$ on the reaction given in (5).

The particular type of the reaction schemes considered here (3) is inspired by chemical processes in the living cell. It is often that by action of enzymes certain input set of chemicals is converted into output molecules by series of intermediate reactions. It is exactly this aspect of cellular machinery which this scheme tries to capture in the most simple way. The most general reaction graph one can consider is shown in Fig. 2 The graph indicates that cell machinery converts molecules $X_{1}, \ldots, X_{\omega}$ into molecules $X_{1}^{\prime}, X_{2}^{\prime}, \ldots, X_{\eta}^{\prime}$. The shaded area in the middle denotes intermediate reaction steps that involve arbitrary set of reactions between molecules already shown in the graph. An additional particle types may appear in the shaded region. It is possible to define the speed of a reaction as the time needed for the predetermined set of output molecules $X_{1}^{\prime}, X_{2}^{\prime}, \ldots, X_{\eta}^{\prime}$ to appear for the first time, provided only the input molecules were present initially in the system. How this idea is implemented is shown bellow.

In addition to reaction scheme given in Eq. (3) two quantities are specified. First, at $t=0$ a certain number of particles is injected into the system in various containers. The list of the particles injected is specified by the vector

$$
\boldsymbol{\iota}=\left(n_{1,1}^{(0)}, \ldots, n_{\alpha, i}^{(0)}, \ldots, n_{M, N}^{(0)}\right)
$$

In the course of time the injected set of particles will be transformed into something else. Second, the vector

$$
\boldsymbol{\pi}=\left(n_{1,1}^{*}, \ldots, n_{\alpha, i}^{*}, \ldots, n_{M, N}^{*}\right)
$$

specifies tasks that have to be achieved. For example, $n_{\alpha, i}^{*}>0$ indicates that goal is to synthesize $n_{\alpha, i}^{*}$ molecules of the type $X_{\alpha}$ in the container $C_{i}$. On the other hand, $n_{\alpha, i}^{*}=0$ indicates that the number of particles $X_{\alpha}$ in the container $C_{i}$ is not traced. The maximum number of tasks is given by $N \times M$, though not all tasks need to be monitored. Also, the cases where task is achieved trivially at $t=0$ are forbidden and additional restriction is set upon components of $\boldsymbol{\iota}$ and $\boldsymbol{\pi}$ : If task is traced one has $n_{\alpha, i}^{*}>0$. In such case, the condition $n_{\alpha, i}^{*}>n_{\alpha, i}^{(0)}$ must hold.

To make a notation easier, it is convenient to eliminate the elements from $\iota$ and $\boldsymbol{\pi}$ that are zero and write

$$
\boldsymbol{\iota}=\left(\iota_{1}, \iota_{2}, \ldots, \iota_{\omega}\right), \quad \boldsymbol{\pi}=\left(\pi_{1}, \pi_{2}, \ldots, \pi_{\eta}\right)
$$

where $\boldsymbol{\iota}$, and $\boldsymbol{\pi}$, only contain list of molecules injected, and tasks that are actually monitored. It is clear that $\omega, \eta \leq M \times N$.

Every time a certain task is accomplished the time when this happens is stored. These times are arranged in the vector

$$
\mathcal{T}=\left(\mathcal{T}_{1}, \mathcal{T}_{2}, \ldots, \mathcal{T}_{\eta}\right)
$$

and there is a one to one correspondence between the elements of $\boldsymbol{\pi}$ and $\mathcal{T}$. Once the task is achieved molecules that were used to accomplish it are removed from the system. This consideration is motivated by the character of the real processes in the living cell. If certain number of molecules are needed at specific place, once arriving there, these molecules will be consumed by other biochemical processes in the living cell.

The vector $\mathcal{T}$ is a stochastic variable and can be described in terms of the distribution function $\Phi\left(t_{1}, t_{2}, \ldots, t_{n} ; \iota, \pi\right)$. In practise, it is very hard to obtain full distribution function and it is more convenient to use first two moments, the average

$$
\boldsymbol{\tau}=\left(\tau_{1}, \ldots, \tau_{k}, \ldots, \tau_{n}\right)
$$

and the variance

$$
\boldsymbol{\sigma}=\left(\sigma_{1,1}, \ldots, \sigma_{\alpha, i}, \ldots, \sigma_{M, N}\right)
$$

In addition, one could also include moments of the type $\left\langle\tau_{i} \tau_{j}\right\rangle i \neq j$ but these will be not considered.

The quadruple consisting of particular reaction scheme $(\lambda, \xi, \boldsymbol{\Lambda}$ and $\boldsymbol{K})$, network geometry $\left(l_{i, j} i, j=1, \ldots, N\right)$, inject pattern $\boldsymbol{\iota}$, and list of tasks monitored $\boldsymbol{\pi}$, will be referred to as an organism. The organism can be seen as the entity that has to transform a certain number of chemicals into a set of molecules that have to be synthesized at certain places, utilizing available reaction scheme 
and geometry. In the following sections various organisms will be classified according to the criteria how fast they achieve certain list of tasks (see. Eq. [10). In principle, it is possible to make classification with regard to how noisy performance is (see Eq. 11), but this type of classification is left for future studies.

\section{THE DYNAMICS}

The dynamics of the system defined in the previous section is stochastic and from the rules discussed one can derive a master equation that describes the time evolution of the occupation probabilities $p(\boldsymbol{c}, t)$,

$$
\dot{p}(\boldsymbol{c}, t)=\sum_{\boldsymbol{c}^{\prime}} R_{\boldsymbol{c}, \boldsymbol{c}^{\prime}} p\left(\boldsymbol{c}^{\prime}, t\right)-\sum_{\boldsymbol{c}^{\prime}} R_{\boldsymbol{c}^{\prime} \boldsymbol{c}} p(\boldsymbol{c}, t)
$$

where here and in the following dot over symbol denotes time derivative. The reaction rates $R_{\boldsymbol{c}^{\prime} \boldsymbol{c}}$ describing transition $\boldsymbol{c} \rightarrow \boldsymbol{c}^{\prime}$ can be easily calculated from the definition of the model. In general, it is very hard to solve the equation (12). In here, two strategies are use to solve it. First strategy is based on a simulation method. This is a straight forward approach. In the second instance a set of equations is derived that specifies first, second, and possibly higher moments of the $\Phi\left(t_{1}, \ldots, t_{n} ; \iota, \pi\right)$. Both strategies are implemented into a computer program.

Computer Simulation: The master equation (12) is solved by using minimal process algorithm suggested by Gillespie [54, 55. Given that the system is in a certain configuration, one can calculate distribution of waiting time for the next process to happen. Time is updated by amount of waiting $\Delta t$. Process is chosen randomly according to weight given by corresponding rates for each process. For problem at hand a linear selection algorithm is used.

Given that at the time $t$ the system was in the conformation $\boldsymbol{c}$ specified in (1), following processes can happen. Transport of particle $X_{\alpha}$ from $C_{i}$ to $C_{j}$ occurs with rates

$$
R_{i, j}^{\alpha}(\boldsymbol{c})=D_{i, j}^{\alpha} n_{\alpha, i}, \quad \alpha=1,2, \ldots, M, \quad i, j=1, \ldots, N
$$

or reactions within containers with rates

$$
R_{\alpha, \beta}^{i}(\boldsymbol{c})=\lambda_{\alpha, \beta}^{i}\left(\boldsymbol{n}_{i}\right) n_{\alpha, i}
$$

One also needs the total reaction rate,

$$
Q(\boldsymbol{c})=\sum_{i, j=1}^{N} \sum_{\alpha=1}^{M} R_{i, j}^{\alpha}(\boldsymbol{c})+\sum_{i=1}^{N} \sum_{\alpha, \beta=1}^{M} R_{\alpha, \beta}^{i}(\boldsymbol{c})
$$

which is used to calculate the time update $\Delta t=-\ln (1-$ $r) / Q$ where $r$ is a random number drawn uniformly from the interval $[0,1)$. The probabilities for process to happen are given by

$$
p_{i, j}^{\alpha}=\frac{R_{i, j}^{\alpha}(\boldsymbol{c})}{Q}, \alpha=1, \ldots, M, i, j=1, \ldots, N
$$

$$
p_{\alpha, \beta}^{i}=\frac{R_{\alpha, \beta}^{i}(\boldsymbol{c})}{Q}, i=1, \ldots, N, \alpha, \beta=1, \ldots, M
$$

A process is chosen using the linear selection algorithm. First, a new random number $r^{\prime}$ is drawn. After that, the cumulant probabilities are calculated through loop over all processes. The loop is stopped when the cumulant probability exceeds $r^{\prime}$ and the last process for which this happened is executed.

Moment method: This method relies on the matrix representation of the master equation (12) and can not be used to treat cases with large number of containers and particle types. However, the method is exact and should be used when there are enough computation resources. The method is developed in the following section.

\section{THE CALCULATION OF THE AVERAGE AND STANDARD DEVIATION}

It will be shown how to calculate moments of the individual components of $\boldsymbol{\tau}, \gamma_{k}^{(p)} \equiv\left\langle\left(\tau_{k}\right)^{p}\right\rangle, k=1, \ldots, \eta$ and $p=0,1,2, \ldots, \infty$. The more complicated moments, e.g. $\gamma_{k, l}^{(p, q)} \equiv\left\langle\left(\tau_{k}\right)^{p}\left(\tau_{l}\right)^{q}\right\rangle$ with $k, l=1, \ldots, \eta$ and $p, q=0,1,2, \ldots, \infty$, could be also evaluated using technique presented in this section, but such moments will not be considered. The focus in on the moments that are obtained through

$$
\gamma_{k}^{(p)}(\boldsymbol{\iota}, \boldsymbol{\pi})=\int_{0}^{\infty} t^{p} \Gamma_{k}(t ; \boldsymbol{\iota}, \boldsymbol{\pi}) d t
$$

where $\Gamma_{k}(t ; \boldsymbol{\iota}, \boldsymbol{\pi})$ denotes integrated distribution function for task $\pi_{k}$ given by

$$
\Gamma_{k}(t ; \iota, \boldsymbol{\pi}) \equiv \int_{0}^{\infty} \Phi\left(t_{1}, \ldots, t_{n} ; \iota, \boldsymbol{\pi}\right) \prod_{m=1, \eta}^{m \neq k} d t_{m}
$$

Please note that accomplishment of each individual task is influenced by presence of others since the particles can vanish upon accomplishment of various tasks. This is the reason why integrated distribution function $\Gamma_{k}(t ; \boldsymbol{\iota}, \boldsymbol{\pi})$ contains both index of the task that statistics is sought for $(k)$ and full list of tasks being monitored $(\boldsymbol{\pi})$.

It is possible to find closed expression for Laplace transform of $\Gamma_{k}(t ; \boldsymbol{\iota}, \boldsymbol{\pi})$. The Laplace transform of arbitrary function $F(t)$ is defined as $F(s) \equiv$ $\int_{0}^{\infty} \exp (-s t) F(t) d t . \Gamma_{k}(s ; \boldsymbol{\iota}, \boldsymbol{\pi})$ is given by

$$
\begin{array}{r}
\Gamma_{k}(s ; \boldsymbol{\iota}, \boldsymbol{\pi})=\sum_{\boldsymbol{c} \neq \boldsymbol{\iota}} w\left(\boldsymbol{c}, \pi_{k}\right) g(s ; \boldsymbol{\iota}, \boldsymbol{c}, \boldsymbol{\pi}) \\
+\sum_{m=1, \eta} \sum_{\boldsymbol{c} \neq \boldsymbol{\iota}} w\left(\boldsymbol{c}, \pi_{m}\right) g(s ; \boldsymbol{\iota}, \boldsymbol{c}, \boldsymbol{\pi}) \\
\times \Gamma_{k}\left(s ; \boldsymbol{c} / \pi_{m}, \boldsymbol{\pi} / \pi_{m}\right)
\end{array}
$$


where notation used is as follows. The $w\left(\boldsymbol{c}, \pi_{k}\right)$ equals one if task $\pi_{k}$ can be accomplished once system arrives in the state $\boldsymbol{c}$, and equals zero otherwise. In the following, by definition, a state for which one of the $w\left(\boldsymbol{c}, \pi_{k}\right)$ with $k=1, \ldots, \eta$ differs from zero will be referred to as a window state. Through window states tasks can be accomplished. $\boldsymbol{c} / \pi_{m}$ denotes the state immediately after the particles have been taken away once the task $\pi_{m}$ was accomplished. Likewise, the symbol $\boldsymbol{\pi} / \pi_{m}$ denotes a list of tasks being monitored with task $\pi_{m}$ omitted $\left(\pi_{1}, \ldots, \pi_{m-1}, \pi_{m+1}, \ldots, \pi_{\eta}\right) . \quad g(s ; \boldsymbol{\iota}, \boldsymbol{c}, \boldsymbol{\pi})$ is a distribution function for the first passage time into the state $c$ given that the dynamics started from the state $\iota$. Fig. [3 is a schematic presentation of the Eq. (20). Please note that Eq. (20) defines $\Gamma_{k}(s ; \boldsymbol{\iota}, \boldsymbol{\pi})$ recursively. The number of tasks on the right hand side of Eq. (20) is by one smaller than the same number on the left hand side of the equation. When list of a tasks is empty $\boldsymbol{\pi}=\boldsymbol{\pi}_{0}$ and $\boldsymbol{\pi}_{0} \equiv()$. Condition $\Gamma_{k}\left(s ; \boldsymbol{\iota}, \boldsymbol{\pi}_{0}\right)=0$ stops recursion.

The Laplace transform of the first arrival time distribution function $g(t ; \boldsymbol{\iota}, \boldsymbol{c}, \boldsymbol{\pi})$ is calculated as follows. Given the particular reaction scheme it is possible to construct master equation (12) that governs the time dependence of the occupation probabilities of each state $p(\boldsymbol{c}, t)$, where $\boldsymbol{c}$ has to be accessible from the initial state $\boldsymbol{\iota}$. When calculating matrix of the transition rates $\boldsymbol{R}$ it is assumed that all window-states can not be left once they are arrived into. Window states are perfectly absorbing. Fig. [3] is a graphic representation of this fact. Once $p(\boldsymbol{c}, t)$ is found from (12) the first passage time distribution function is given by $g(t, \boldsymbol{\iota}, \boldsymbol{c}, \boldsymbol{\pi})=\dot{p}(\boldsymbol{c}, t)$.

It is useful to arrange both $p(\boldsymbol{c}, t)$ and $g(s ; \boldsymbol{\iota}, \boldsymbol{c}, \boldsymbol{\pi})$ into a vectors $\boldsymbol{p}(t)$ and $\boldsymbol{g}(t)$ where notation was simplified a bit since we assume that $\iota$ and $\boldsymbol{\pi}$ are known and fixed. It is useful to rewrite master equation (12) in a matrix form as $\dot{\boldsymbol{p}}(t)=\boldsymbol{R} p(t)$. This equation is solved using Laplace transform, with initial condition $p(\boldsymbol{c}, 0)=\delta_{\boldsymbol{c}, \boldsymbol{\iota}}(\delta$ denotes Kronecker delta symbol): $s \boldsymbol{p}(s)-\boldsymbol{p}_{0}=\boldsymbol{R} p(s)$. Also, in the Laplace transform space one has $s \boldsymbol{p}(s)-\boldsymbol{p}_{0}=\boldsymbol{g}(s)$, which directly leads to the equation for the first arrival time distribution function:

$$
s \boldsymbol{g}(s)=\boldsymbol{R}\left[\boldsymbol{g}(s)+\boldsymbol{p}_{0}\right]
$$

In principle, the equation above could be solved as $\boldsymbol{g}(s)=$ $(s-\boldsymbol{R})^{-1} \boldsymbol{p}_{0}$. However, matrix $\boldsymbol{R}$ has zero eigenvalues and the value of $\boldsymbol{g}(s)$ in the limit $s \rightarrow 0$ is ill defined. To avoid such problems Eq. (21) has to be solved in a special way.

It is useful to separate configuration space into three groups, as shown in Fig. 3 First group, labeled $S_{n}$, contains states that are non-window or the normal-states. Second group contains states labeled by $S_{t}$ that we refer to as the trap states. The third group consists of the window states solely, labeled by $S_{w}$. The existence of the trap states is problem dependent. Once system arrives into these states there is no exit from this space, though such states are not window states. This simply means that it is possible that set of tasks is never accomplished.
Using the partition of states shown in Fig. 3 leads to the following set of equations

$$
\begin{aligned}
& s \boldsymbol{g}_{n}(s)=\boldsymbol{R}_{n n}\left[\boldsymbol{g}_{n}(s)+\boldsymbol{p}_{n, 0}\right] \\
& s \boldsymbol{g}_{t}(s)=\boldsymbol{R}_{t n}\left[\boldsymbol{g}_{n}(s)+\boldsymbol{p}_{n, 0}\right]+\boldsymbol{R}_{t t} \boldsymbol{g}_{t}(s) \\
& s \boldsymbol{g}_{w}(s)=\boldsymbol{R}_{w n}\left[\boldsymbol{g}_{n}(s)+\boldsymbol{p}_{n, 0}\right]
\end{aligned}
$$

Please note that $\boldsymbol{p}_{t, 0}$ and $\boldsymbol{p}_{w, 0}$ are zero since, initially, the system is in the state $\iota$ and such state does not have any components in the $S_{t}$ and $S_{w}$ spaces. Given that there are no transition from trap states into normal states or window states blocks $\boldsymbol{R}_{n t}$ and $\boldsymbol{R}_{w t}$ are missing in the equations above. Likewise, blocks $\boldsymbol{R}_{w w}, \boldsymbol{R}_{n w}$ and $\boldsymbol{R}_{t w}$ and are zero since there are no transitions among window states, nor transitions from them.

The solution of the equations (22)-(24) can be found in a straight forward manner. Equation (22) can be solved first, leading to $\boldsymbol{g}_{n}(s)=\left(s-\boldsymbol{R}_{n n}\right)^{-1} \boldsymbol{p}_{n, 0}$ and inserting this expression into the Eq. (24) gives

$$
g(s, \boldsymbol{\iota}, \boldsymbol{c}, \boldsymbol{\pi})=\left[\boldsymbol{R}_{w n}\left(s-\boldsymbol{R}_{n n}\right)^{-1} \boldsymbol{p}_{n, 0}\right]_{\boldsymbol{c}}, \quad \boldsymbol{c} \in S_{w}
$$

Please note that Eq. (25) is well defined for all values of s. In particular, in the limit $s \rightarrow 0$ even for a matrix $\boldsymbol{R}$ that has zero eigenvalues. It is intuitively clear that, contrary to $\boldsymbol{R}$, matrix $\boldsymbol{R}_{n n}$ does not have zero eigenvalues: as time goes on, all probability accumulates in $S_{w}$ and $S_{t}$ spaces (see Fig. 3). The only difficulty with Eq. (25) is partitioning of the full configuration space into $S_{n}, S_{w}$ and $S_{t}$. The algorithm for carrying out such partitioning is not presented here in order to save the space.

Finally, once $g(s, \boldsymbol{\iota}, \boldsymbol{c}, \boldsymbol{\pi})$ is found one can proceed with the calculation of the moments $\gamma_{k}^{p}(\boldsymbol{\iota}, \boldsymbol{\pi})$. These can be obtained by taking derivatives of Eq. (20) with regard to $s$ and setting $s=0$ at the end: it can be seen easily from Eq. (18) that

$$
\gamma_{k}^{p}(\boldsymbol{\iota}, \boldsymbol{\pi})=(-)^{p} \lim _{s \rightarrow 0} \partial_{s}^{p} \Gamma_{k}(s, \boldsymbol{\iota}, \boldsymbol{\pi})
$$

where $\partial_{s}$ denotes derivative over $s$. Using Eqs. (26) and (20) leads to

$$
\begin{aligned}
& \gamma_{k}^{(p)}(\boldsymbol{\iota}, \boldsymbol{\pi})= \sum_{\boldsymbol{c} \neq \boldsymbol{\iota}} w\left(\boldsymbol{c}, \pi_{k}\right) g^{(p)}(\boldsymbol{\iota}, \boldsymbol{c}, \boldsymbol{\pi}) \\
&+\sum_{m=1, \eta}^{m \neq k} \sum_{\boldsymbol{c} \neq \boldsymbol{\iota}} w\left(\boldsymbol{c}, \pi_{m}\right) \sum_{q=0, p}\left(\begin{array}{c}
p \\
q
\end{array}\right) g^{(p)}(\boldsymbol{\iota}, \boldsymbol{c}, \boldsymbol{\pi}) \\
& \quad \times \gamma_{k}^{(p-q)}\left(\boldsymbol{c} / \pi_{m}, \boldsymbol{\pi} / \pi_{m}\right)
\end{aligned}
$$

where by definition $g^{(p)}(\boldsymbol{\iota}, \boldsymbol{c}, \boldsymbol{\pi})$ $(-)^{p} \lim _{s \rightarrow 0} \partial_{s}^{p} g(s, \boldsymbol{\iota}, \boldsymbol{c}, \boldsymbol{\pi})$, which after using Eq. (25) leads to

$$
g^{(p)}(\boldsymbol{\iota}, \boldsymbol{c}, \boldsymbol{\pi})=(-)^{(p+1)} p !\left[\boldsymbol{R}_{w n} \boldsymbol{R}_{n n}^{-(p+1)} \boldsymbol{p}_{n, 0}\right]_{\boldsymbol{c}}
$$

Equations (27)-(28) are central result of this section. They determine all moments. For example, once 
$\gamma_{k}^{(p)}(\boldsymbol{\iota}, \boldsymbol{\pi}) p=0,1,2$ are found the average and variance of $\mathcal{T}$ are given by

$$
\begin{aligned}
\tau_{k}(\boldsymbol{\iota}, \boldsymbol{\pi}) & =\frac{\gamma_{k}^{(1)}(\boldsymbol{\iota}, \boldsymbol{\pi})}{\gamma_{k}^{(0)}(\boldsymbol{\iota}, \boldsymbol{\pi})} \\
\sigma_{k}(\boldsymbol{\iota}, \boldsymbol{\pi})^{2} & =\frac{\gamma_{k}^{(2)}(\boldsymbol{\iota}, \boldsymbol{\pi})}{\gamma_{k}^{(0)}(\boldsymbol{\iota}, \boldsymbol{\pi})}-\left[\frac{\gamma_{k}^{(1)}(\boldsymbol{\iota}, \boldsymbol{\pi})}{\gamma_{k}^{(0)}(\boldsymbol{\iota}, \boldsymbol{\pi})}\right]^{2}
\end{aligned}
$$

where $k=1, \ldots, \eta$. One has to divide by $\gamma_{k}^{(0)}(\boldsymbol{\iota}, \boldsymbol{\pi})$ in the equations above in order to ensure that in the case when there is a possibility that some tasks are not accomplished statistics is done only for instances where task was achieved. The percentage of cases when this happened is given by $\gamma_{k}^{(0)}(\boldsymbol{\iota}, \boldsymbol{\pi})$. The numerical implementation of Eqs. (27) and (28) is a straight forward and gives the exact values for $\boldsymbol{\tau}$ and $\boldsymbol{\sigma}$.

\section{ROGE ENSEMBLE: DESCRIBING ORGANISM PERFORMANCE IN TERMS OF THE SINGLE VARIABLE $\nu$}

The calculation of $\boldsymbol{\tau}$ and $\boldsymbol{\sigma}$ for an arbitrary organism was discussed in the previous section. In this section the GORE ensemble will be studied. The case $N=1$ where there is only one container is not interesting since such space is not structured. The first non-trivial example of a structured space is the case of a two-container network with $N=2$. For simplicity reasons, a situation will be considered where there are only two particle types $\mathrm{A}$ and B corresponding to $M=2$. With only one particle type one can only focus on transport issues. To see coupling between reactions and transport one needs at least two particles types. Please note that the analysis done in this section is quite generic, though it is carried out on a rather simple case of $N=2$ and $M=2$. The analysis could be easily repeated for an arbitrary values of $N$ and $M$. However, the computational cost scales with $N$ and $M$, and there is clearly an upper limit to which cases one can study. It will be shown that the relatively simple twocontainer network and reactions with two particle types provide interesting insight into the problem.

The structure of the organisms in the ROGE ensemble is defined as follows. For each organism in the ensemble an unique choice is made for (i) total number of $\mathrm{A}$ and $\mathrm{B}$ molecules, (ii) reactivity matrix $\boldsymbol{\Lambda}$, (iii) catalytic activity matrix $\boldsymbol{K}$, (iv) inject pattern $\boldsymbol{\iota}$ and (v) list of tasks monitored $\boldsymbol{\pi}$. For all organisms geometry is kept fixed (e.g. size of containers and length of the tube connecting them). Please note that there are two symmetries in the problem, the one that originates from relabeling of particles, and another one that has to do with relabeling of containers. A special care is taken to eliminate these symmetries in the ensemble. The goal is to unearth best reaction scheme (organism) that draws most benefit from the structured space that has the form of a two-container network.

We start with the situation where the total number of particles $N_{p}^{*}=n_{A, 1}+n_{A, 2}+n_{B, 1}+n_{B, 2}$ in the system equals one. Also, we consider only organisms with reactions $A \rightarrow B$ and $B \rightarrow A$, both with rate $\lambda$. One can see that, for a given reaction scheme, and with a constraint $N_{p}^{*}=1$, only three choices for $\iota$ and $\boldsymbol{\pi}$ are possible, leading to the three organisms $o_{1}, o_{2}$ and $o_{3}$ that are listed in table 【 Please note that all other $N_{p}^{*}=1$ cases can be related to these three through relabeling of particles and containers, or by considering different reaction schemes.

All organisms are such that one A particle is injected in the container $C_{1}$ and only one task is monitored. In the case of organism $o_{1}$ the goal is to synthesize one A molecule in the container $C_{2}$, in the case of $o_{2}$ one $\mathrm{B}$ molecule should be synthesized in $C_{1}$, while in the case of $o_{3}$ one B molecule should be created in $C_{2}$. Reaction process is stochastic. Average and standard deviation of time for task accomplishment are given under columns labeled $\tau$ and $\sigma$ in table \ The dependence of $\tau$ and $\sigma$ on (the inverse of) the transport rate $D$ is depicted in

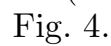

Clearly, all three organisms achieve their tasks faster

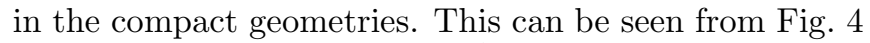
since $\tau$ gets smaller when $D^{-1}, l \rightarrow 0$. (Here, we used the fact that $D^{-1} \sim l^{2}$ where $l$ is the length of the tube connecting containers). Same holds for curves depicting $\sigma$. Amount of noise decreases when geometry is compact. For example, organism $o_{1}$ functions by transporting one A particle from container $C_{1}$ to container $C_{2}$. It is intuitively clear that this happens faster when containers are close. One can analyze $o_{3}$ in the similar way. However, $\mathrm{O}_{2}$ is somewhat different. In the case of $o_{2}$ the goal is to synthesize one $\mathrm{B}$ molecule in the same container where A was injected. Figure 4 illustrates the fact that when another open volume is present molecule can wonder away into additional volume and this process delays synthesis of B. One can also see that $o_{2}$ is least sensitive to increase (decrease) in $l(D)$ : when $l, D^{-1} \rightarrow \infty$ the $\tau$ for $o_{1}$ and $o_{3}$ increases, while for $o_{2} \tau$ saturates to constant (though level of noise, $\sigma$, increases).

The question is whether it is possible to present information conveyed from Fig. \and table $\square$ in a more compact way? There are couple of reasons for this. First, in a case of the more complicated geometry, generating

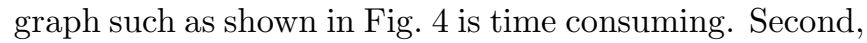
it would be desirable to have automatic procedure for assessing most important properties of such graph; whether given organism performs best in compact or structured (network like) geometry.

To achieve this goal and compactify information in Fig. प and table $\prod_{\text {it }}$ is useful to consider following quan- 
tity,

$$
\nu=\frac{\left\|\tau_{n}\right\|}{\left\|\tau_{0}\right\|}
$$

where $\|$.$\| denotes Euclidean norm of the vector, \boldsymbol{\tau}_{n}$ and $\boldsymbol{\tau}_{0}$ are given by $\boldsymbol{\tau}$ calculated for network like and compact geometries with jump rates $D_{n}$ and $D_{0}$ such that $D_{n} \sim$ $\lambda$ and $D_{0} \gg D_{n}, \lambda, \xi \lambda$. Using similar reasoning the $\nu$ can be defined for generic network having more than two containers. In such a way one can compare extended and compact geometries of given fixed network structure and express comparison through one variable $\nu$.

In the following the $\nu$ will be refereed to as speed of a reaction. $\nu<1$ is indication that organisms accomplishes tasks faster in a network like geometry, while $\nu>1$ shows that organism draws most benefit from a compact geometry. Please note, all organisms considered in table $\prod$ and Fig. 4 have $\nu>1$.

In principle, one could define quantity similar to $\nu$ and use $\sigma$ instead of $\tau$. For example, one could use

$$
\mu=\frac{\left\|\sigma_{n}\right\|}{\left\|\sigma_{0}\right\|}
$$

where meanings of $\boldsymbol{\sigma}_{n}$ and $\boldsymbol{\sigma}_{0}$ are similar to the ones of $\boldsymbol{\tau}_{n}$ and $\boldsymbol{\tau}_{0} . \mu$ could be used to classify organisms in the ensemble according to the amount of noise in compact and extended (network-like) geometries. Also, it is possible to use $\rho=\sqrt{\nu^{2}+\mu^{2}}$ as simultaneous measure of speed and noise. But such quantities will not be studied at the moment. From now on we focus on $\nu$ solely.

\section{ROGE ENSEMBLE: CLASSIFICATION OF THE REACTION SCHEMES USING THE SPEED OF REACTION $\nu$}

Figure 5 depicts results of the classification of large number of organisms in five ROGE ensembles where $\nu$ (defined in previous section) is used as a measure of the performance. Five ROGE ensembles are constructed with the increasing upper limit for the total number of particles in the system $N_{p}^{*}$ from 1 to 5 .

For $N_{p}^{*}=1$ there are 95 unique organisms. Clearly, this number is overestimate. The number of unique organisms is obtained after eliminating symmetries related to relabeling of particles and containers removed. However, this number is still too large since catalytic influences are assumed to play the role, though there can not be any catalytic influence when there is constantly one molecule in the system. The number of unique organisms for other cases discussed later with $N_{p}^{*}=2,3,4,5$ is correct. There are no organisms in the $N_{p}^{*}=1$ class that benefit from the structured geometry since the histogram in the panel (b) is empty. All organisms have $\nu>1$ as can be seen from panel (a).
For $N_{p}^{*}=2$ there are 730 organisms that are unique. Only after more than one molecule appears in the system, catalytic influences start to play the role, and organisms that benefit from the network like structure appear [see

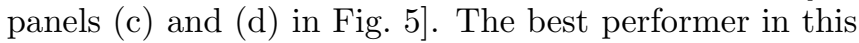
class is given in Table III denoted by $o_{i, 2}$, together with couple of a second best performers denoted by $o_{i i, 2}$. The two organisms labeled $o_{i, 2}$ draw most benefit from the network like structure and degeneracy in $\nu$ comes from the fact that same enhancement and reduction factor is used for positive and negative catalytic influence respectively.

One can understand intuitively why $o_{i, 2}$ runs faster in the network like geometry. We focus on the particular case of $o_{i, 2}$ where goal is to synthesize two B molecules in the container $C_{1}$. Due to the initial presence of molecule A in the container $C_{1}$, it is very likely that $\mathrm{B}$ molecule will be converted into A molecule, when $\mathrm{A}$ and $\mathrm{B}$ meet in the same container. Once there are two A molecules in the system the trouble starts. Even if the reaction $A \rightarrow B$ happens, and chance for this is really small due to the negative catalytic influence of $\mathrm{A}$ on such reaction, $\mathrm{B}$ will be converted back to $\mathrm{A}$ immediately (due to the positive catalytic influence of another A molecule on the $B \rightarrow A$ reaction). In principle, conversion of $\mathrm{AB}$ into $2 \mathrm{~B}$ has no chance occurring in a reasonable time when there is only one container. The antagonistic catalytic influences just discussed are best handled in a network like geometry. In such a case the synthesis of the molecules can be done in a separate containers. With two containers there is always a chance that antagonistic influences will be reduced. For example, in the case there are two A molecules in the system, the processes $A \rightarrow B$ can happen fast given that damage inflicting A molecule is in another container.

Also, it is naive to think that organisms containing reactions with solely negative catalytic influence perform best in a network like structure. This is clearly not the case. The winning organisms $o_{i, 2}$ are constructed from one reaction with positive $B \stackrel{+A}{\longrightarrow} A$ and another reaction $A \stackrel{-A}{\longrightarrow} B$ with negative catalytic influence. Furthermore, Fig. 5 shows plenty of other cases. Actually, the completely opposite is possible. There are many organisms in the histogram plot with $\nu>1$ that contain at least one reaction with negative catalytic influence and these organisms perform best in a compact geometry.

With $N_{p}^{*}=3$ there are 3025 organisms that are unique. The best performer in this class is given in Table III denoted by $o_{i, 3}$. A couple of the second best performers are also shown and labeled $o_{i i, 3}$. When more than two molecules appear in the system, completely new organism appears as winner. There is a sharp transition in character of winners. Both the reaction type and inject and task patterns are different in $o_{i, 3}$ and $o_{i, 2}$. The reaction of organisms $o_{3, i}$ is such that it is possible that tasks are not accomplished. For example, all A molecules can be converted to B before task [2A]-[ ] in $C_{1}$ is accom- 
plished. Once this happens synthesis of A's in $C_{1}$ will never occur since back reaction $B \rightarrow A$ is absent.

Very likely, from biological point of view there is no advantage basing survival on a reaction that sometimes fails, i.e. using reaction similar to the one contained in $o_{i, 3}$. Nevertheless, there might be constraints that could enforce presence of such reaction. For example, the number of molecules in Nature is large but limited. Living organisms have to use what is available. In the lack of alternatives it might be necessary for the intra cellular machinery to use reaction that sometimes fails. But leaving such discussions aside, the only point emphasized here is that organism $o_{i, 3}$ draws most benefit from the network like structure.

Interestingly, this type of organism remains winner in the classes $N_{p}^{*}=4$ and $N_{p}^{*}=5$ with $\nu=0.0248$ and $\nu=0.0192$, see table 1 organisms $o_{i, 4}$ and $o_{i, 5}$. However, please note that details of the inject pattern of $o_{i, 4}$ and $o_{i, 5}$ are somewhat different from $o_{i, 3}$. Judging solely from $o_{i, 3}$ and $o_{i, 4}$ one would guess that inject patter for best performer in class $N_{p}^{*}=5$ should be $\iota=[\mathrm{A}]-[4 \mathrm{~A}]$, in class $N_{p}^{*}=6 \iota=[\mathrm{A}]-[5 \mathrm{~A}]$, etc. However, this is not the case. The best performer in class $N_{p}^{*}=5$ has inject pattern equal to $\iota=[2 \mathrm{~A}]-[3 \mathrm{~A}]$ with $\nu=0.0192$, while organism with $\iota=[\mathrm{A}]-[4 \mathrm{~A}]$, denoted by $o_{i i, 2}$, has somewhat larger $\nu=0.0219$.

Thus, examples above show how difficult it is to have any intuition about structure of best performers. There are also other ways to see this. For example, the last row in table contains organisms with slightly modified inject or task patterns where original form is taken from best performer. Comparing $o_{i, 3}$ and $o_{*, 3}, o_{i, 4}$ and $o_{*, 4}$, and finally $o_{i, 5}$ with $o_{*, 5}$ shows that small alternation in task pattern, obtained by moving one $\mathrm{B}$ particles from $C_{2}$ into $C_{1}$, lowers performance considerably. Organisms with such alternations do not perform well in the network like geometry: each of $o_{*, 3}, o_{*, 4}$ and $o_{*, 5}$ has $\nu>1$. Another example, can be obtained from comparison of $o_{i, 3}$ with $o_{*, 2}$. Both inject and task patterns have been altered in $o_{i, 3}$. This change is motivated by sequence of organisms in first row of table III when read from right to left. A priori, the organism $o_{*, 2}$ could be considered to have $\nu<1$, however this is not the case. The actual value for $\nu=418$ is (lot) larger than one.

In summary, the table II shows a couple of interesting features. First, one can see that when maximum allowed number of particles in the system increases new effects appear. It is impossible to predict winner in each class before calculation is done. Second, there is also a large sensitivity on inject and task patterns. Slight alternation of these patterns can lead to drastic changes in performance criteria $\nu$.

\section{DISCUSSION}

We introduced what we might call a generic model for study of chemical reactions in structured spaces, based on a simple way of incorporating interplay between transport, chemical reactions, and geometry. A number of different chemical reactions were studied and their performance compared in various ways. The main idea is to see how the reaction processes behave when geometry changes from compact open space towards the cell like environment that is more structured. In order to be able to analyze large number of reactions, relatively simple model was used in order to reduce computational time. Following simplifications were made.

As a model of structured space we used a network consisting of containers connected by tubes. Such setup captures the most important geometrical aspects of the problem. There are compact regions in space that reactants can explore and react within. These regions are connected to each other and exchange chemicals. In such a way the two most important processes are clearly separated, the local dynamics within container, and the transport among containers. This is pretty much how living cell operates. In such a way one can capture most important geometrical aspects of the cell interior.

The reaction scheme considered is rather crude. Dynamics within container, however complicated it may be, is projected onto a relatively low number of degrees of freedom, the number of particles in each container. The reaction rate $\lambda$ describes how fast reactions happen. The description in terms of number of particles becomes more and more valid when size of the reaction volume is reduced and number of reactants few [41, 42]. It is exactly this limit that we focussed on.

The transport between containers was modeled in simplest possible way by using the effective transport rate $D$. It was assumed that transport rate is same for any pair of containers, and type of particles. This assumption could be easily relaxed. Clearly, when tubes are long, the number of particles in each container decays non-exponentially and the transport process can not be described by using a transport rate. But such effects are not considered here.

Thus, only two parameters are used to describe dynamics, the reaction rate $\lambda$ and transport rate $D$. There are several advantages in doing so, but the most important one is that reactions and transport are clearly separated, and it is easier to understand how they influence each other. Despite apparent simplicity, the model studied in here captures all essential features of the problem. Should there be need for that one can make the model more realistic, but we refrain from this at the moment.

Which types of chemical reactions draw most benefit from structured spaces? In an attempt to answer this question two schemes were formulated. In ROGE 
scheme one explores variety of chemical reactions while spatial structure (geometry) is kept fixed. It is the other way around for GORE scheme. At the moment ROGE scheme is studied in a lot more detail. The study of GORE ensemble will be left for the future work. The main findings of this work are discussed bellow.

Since dynamics is stochastic one needs probabilistic description of the system, and in order to perform analysis of any chemical reaction one has to solve corresponding master equation that describes statistics of events. Two techniques for doing this were used. First, the computer simulation is a straight forward way to analyze master equation (discussed in section III). This technique is not that accurate due to the stochastic spread of data. One needs unrealistically large number of runs in order to gain reasonable accuracy in $\nu$. In here, a novel method of analyzing chemical reaction was developed, with emphasis on the first passage time (see section IV). This method relies on a matrix representation of the master equation. In principle, it is exact up to the numerical errors in carrying out matrix manipulations, such as finding inverses, multiplying matrix with vector etc. In here we developed a equation that describes first passage time distribution function for achieving set of tasks. From this equation one can easily obtain all moments of distribution function and calculate average execution time for reaction and its variance.

It is not so easy to compare two vastly different chemical reactions. A method for doing this was discussed in section [V] There are some obvious difficulties when doing comparison. For example, one might need to compare a situation where number of molecular types involved in reactions are completely different. Also, spatial structures need not be the same, e.g. there might be a need to compare two vastly different network structures. In order to overcome these difficulties one has to use a measure of relative performance. For example, in the case of a ROGE ensemble the execution time for given reaction was compared when geometry is stretched (transport occurs with rate $D_{n} \sim \lambda$ ) and compact (transport with rate $D_{0}$ where $\left.D_{0} \gg D_{n}, \lambda, \xi \lambda\right)$. The ratio $\nu$ of the magnitude of the execution times $\boldsymbol{\tau}_{n}$ and $\boldsymbol{\tau}_{0}$ for stretched (network) and compact geometry is a rough estimate of how well reaction performs in a network like geometry. Instead of comparing organisms directly one compares values $\nu=\left\|\tau_{n}\right\| /\left\|\tau_{0}\right\|$.

Thus, the single variable $\nu$ is sufficient to determine whether a reaction (organism) runs best on the network like $(\nu<1)$ or the compact geometry $(\nu>1)$. The variable $\nu$ was used to classify performance of various organisms in the geometry consisting of two containers connected by tube, with main findings summarized in Fig. [5] and Table

(1) It is obvious that intuition does not help much. One really has to do numerical analysis in order to extract best performer in a given class. For example, the charac- ter of best performer changes quite a bit when number of particles in the system varies. The structure of the winning organism in the first row of table changes in a rather unpredictable manner as upper limit to the total number of particles in the system increases from 2 to 5 . Also, the performance is extremely sensitive to the details of inject and task patterns. It is interesting to speculate whether such sensitivity on total particle number, inject and task patterns is exploited in the intra cellular machinery. We developed a quantitative method to judge on such effects.

(2) The role of reactions with positive and negative catalytic influence is symmetric. Roughly, they occur equally often in the organisms that perform well in network like geometries with $\nu<1$. Interestingly enough, same holds for opposite range with $\nu>1$. From Fig. 5 one can see that positive and negative areas are roughly equal in magnitude, both in the $\nu<1$ and $\nu>1$ regions. Thus, there are reactions of the suppressor type that run better in compact geometries, and the other way around. There are also reactions with solely positive catalytic influence that run faster in network like geometry. But these findings are the not the only ones that are counterintuitive. It is also surprising that in the region $\nu \approx 0$ reactions with solely positive catalytic influence dominate.

(3) Reaction schemes containing antagonistic catalytic influences in the intermediate stages of reaction, that slow down the production of the final product, require network like geometry to run fast. The antagonistic subreactions have to isolated and allowed to occur in separate regions of space. There are two mechanisms that work against each other. First, the reaction time gets larger since one needs to transport reactants to the regions where damage inflicting sub-reactions must be run in isolation. Second, after being isolated, dangerous subreactions happen much faster than when all reactants are mixed and this shortens reaction time. It is interesting to note that there are large number of cases where isolating misbehaving sub-reactions pays off in faster reaction time.

(4) In general, antagonistic catalytic influences are hard to identify. It is hard to judge reaction by itself. The whole triple consisting of reaction, inject pattern, and task pattern has to be considered simultaneously.

In summary, we studied the workings of a chemical reactions in a two vastly different spaces. In the compact conformation, molecules can reach any part of the space very fast. The transport rate is lot larger than any reaction rate in the system. In the network like conformation, various volumes are well separated and the transport of reactants between volumes occurs relatively slowly. It was found that considerable number of reactions work better in a network like configuration when transport rate gets smaller. The setup suggested in here is generic. There are many possible ways of extending present analysis. For example, at present focus is on 
small number of particles and stochastic dynamics. One could easily consider situation where number of particles is larger and classical chemical kinetics applies in the container. Transport between containers can be treated in a better way. Instead of focusing on the average execution time $\boldsymbol{\tau}$ one can easily look at noise $\boldsymbol{\sigma}$ or combination of the two. The GORE ensemble should be explored in a lot more detail. These issues will be addressed in the future work.

\section{Acknowledgments}

I would like to thank Prof. Owe Orwar and members of his group for fruitful discussions that provided motivation for this work and for warm hospitality during the Särö meeting 2005. The financial support of Prof. Owe Orwar is greatly acknowledged.
[1] G.L. Nelsestuen, Chemistry and Physics of Lipids 101, 37 (1999).

[2] H. Kuthan, Progress in Biophysics \& Molecular Biology 75, 1 (2001).

[3] T. Ganti, BioSystems 7, 15-21 (1975).

[4] M.L. Simpson,G.S. Sayler, J.T. Fleming and B. Applegate, Trends in Biotechnology 19(8), 317 (2001).

[5] A. Mikhailov and B. Hess, J. Phys. Chem. 100, 19059 (1996).

[6] P. Stange, A.S. Mikhailov and B. Hess, J. Phys. Chem. B 102, 6273 (1998).

[7] P. Stange, A.S. Mikhailov and B. Hess, J. Phys. Chem. B 103, 6111 (1999).

[8] P. Stange, D. Zanette, A.S. Mikhailov and B. Hess, Biophysical Chemistry 79, 233 (1999).

[9] P. Stange, A.S. Mikhailov and B. Hess, J. Phys. Chem. B 104, 1844 (2000).

[10] H. Wang, Q. Quyang and Y. Lei, J. Phys. Chem. B 105, 7099 (2001).

[11] K. Sun and Q. Quyang, Phys. Rev. E 64, 026111 (2001).

[12] H. Qian and M. Qian Phys. Rev. Lett. 84(10), 2271 (2000).

[13] M. Calvin, Chemical Evolution: Molecular evolution towards the origin of living systems on the earth and elsewhere, (Claredon Press, Oxford, 1969).

[14] M.V. Volkenstein, Physical Approaches to Biological Evolution, (Springer-Verlag, 1994).

[15] C. Langton, Physica D 22, 120-149 (1986).

[16] C. Furusawa and K. Kaneko, Artificial Life 4, 79-93 (1998).

[17] Z. Vespalcova, A.V. Holden, and J. Brindley, Phys. Lett. A 197, 147-156 (1995).

[18] N. Ono and T. Ikegami, J. Theor. Biol. 206, 243-253 (2000).

[19] M. Boerljist and P. Hogeweg, Physica D 48, 17 (1992).

[20] Karlsson M, Davidson M, Karlsson R, Karlsson A, Bergenholtz J, Konkoli Z, Jesorka A, Lobovkina T, Hurtig J, Voinova M, Orwar O, Ann. Rev. Phys. Chem. 55, 613 (2004).

[21] Karlsson M, Sott K, Davidson M, Cans AS, Linderholm P, Chiu D, Orwar O, Proc. Nat. Acad Sci. USA 99, 11573 (2002).

[22] Karlsson A, Karlsson R, Karlsson M, Cans AS, Stromberg A, Ryttsen F, Orwar O, Nature 409, 150 (2001).

[23] Karlsson M, Nolkrantz K, Davidson MJ, Stromberg A, Ryttsen F, Akerman B, Orwar O, Anal. Chem. 72, 5857 (2000).

[24] J.P. Laplante, M. Pemberton, A. Hjelmfelt and J. Ross, J. Phys. Chem. 99(25), 10063 (1995).
[25] A. Hjelmfelt and J. Ross, J. Phys. Chem. 97, 7988 (1993).

[26] A. Hjelmfelt, F.W. Schneider and J. Ross, Science 260, 335 (1993).

[27] R.J. Bagley and J.D. Farmer, in Artificial Life II, Eds. C.G. Langton, C. Taylor, J.D. Farmer and S. Rasmussen, (Addison-Wesley, 1992), pp. 93-140.

[28] R.J. Bagley, J.D. Farmer and W. Fontana, in Artificial Life II, Eds. C.G. Langton, C. Taylor, J.D. Farmer and S. Rasmussen, (Addison-Wesley, 1992), pp. 141-158.

[29] J.D. Farmer, S.A. Kauffman and N.H. Packard, Physica D 22, 50-67 (1986)

[30] S.A. Kauffman, J. Theor. Biol. 119, 1-24 (1986).

[31] P.F. Stadler, W. Fontana and J.H. Miller, Physica D 63, 378-392 (1993).

[32] P. Schuster, Physica D 22, 100-119 (1986).

[33] F. Slanina and M. Kotrla, Phys. Rev. Lett. 83, 5587 (1999).

[34] S. Jain and S. Krishna, Phys. Rev. Lett. 81, 5684 (1998).

[35] E. Kotomin and V. Kuzovkov, Rep. Prog. Phys. 55, 2079 (1992).

[36] E. Kotomin and V. Kuzovkov, Comprehensive Chemical Kinetics, Vol. 34, R.G.Compton and G. Hancock Editors, (Elsevier, 1996), "Modern aspects of diffusion-controlled reactions".

[37] Comprehensive Chemical Kinetics, Vol. 25, "Diffusionlimited reactions", C.H. Bamford, C.F.H. Tipper and R.G. Compton Editors, (Elsevier, 1985).

[38] E. Kotomin and V. Kuzovkov, Comprehensive Chemical Kinetics, Vol. 34, R.G.Compton and G. Hancock Editors, (Elsevier, 1996), "Modern aspects of diffusion-controlled reactions".

[39] A. S. Mikhailov, Phys. Rep. 184, 307 (1989).

[40] A.A. Ovchinnikov, S.F. Timashev, and A.A. Belyy, "Kinetics of diffusion controlled chemical processses", (Nova Science, 1989).

[41] McQuarrie D, J. Appl. Prob. 4, 413-478 (1967).

[42] Clifford P, Green NJB, Pilling MJ, J. Phys. Chem. 86, 1318-1321 (1982).

[43] R.F. Khairutdinov and N. Serpone, Prog. React. Kinetics 21, 1-68 (1996).

[44] Z. Konkoli, A. Karlsson, and O. Orwar, J. Phys. Chem. B 107, 14077 (2003).

[45] K. Akingbehin, BioSystems 35, 223 (1995).

[46] K. Akingbehin and M. Conrad, Journal of Parallel and Distributed Computing 6, 245 (1989).

[47] M. Conrad, European Journal of Operational Research 30, 280 (1987).

[48] K.G. Kirby and M. Conrad, Physica D22, 205 (1986).

[49] K.G. Kirby and M. Conrad, Bulletin of Mathematical Biology 46(5/6), 765 (1984). 
[50] R. Kampfner and M. Conrad, Bulletin of Mathematical Biology 45(6), 931 (1983).

[51] R. Kampfner and M. Conrad, Bulletin of Mathematical Biology 45(6), 969 (1983).

[52] P. Dittrich, J. Ziegler, and W. Banzhaf, Artificial Life 7, 225-275 (2001).

[53] B. Hess and A. Mikhailov, Science 264, 223 (1994).

[54] D.T. Gillespie, J. Comp. Phys. 22, 403 (1976).

[55] D.T. Gillespie, J. Phys. Chem. 81, 2340 (1977).

Figures

(a)

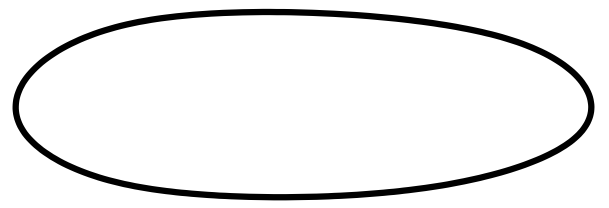

(b)

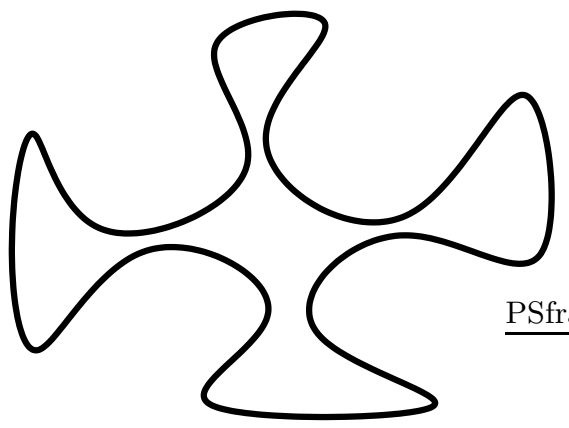

(c)

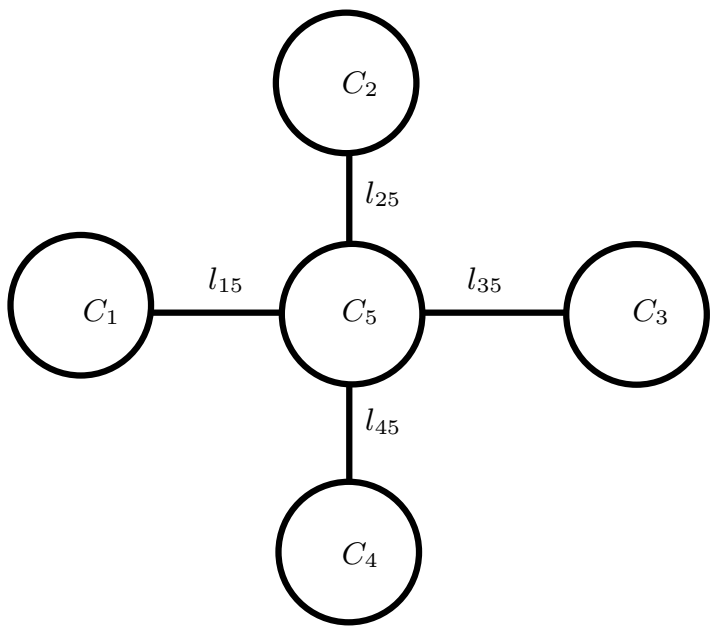

FIG. 1: A guidance how to think about the problem. Panel (a): non-structured (compact) space. Panel (b): structured space obtained by deforming geometry depicted in panel (a). The goal is to understand how reaction dynamics alters when geometry changes from (a) to (b). The panel (c), representing a network of containers and tubes, is used to achieve this goal. For example, to capture the most important geometrical features of the structure in panel (b) one needs five containers $C_{1}, \ldots, C_{5}$ and four tubes with lengths $l_{15}, l_{25}, l_{35}$ and $l_{45}$. The two parameters are used to define the dynamics in the network (c). The intra-container reaction rate $\lambda$, and inter-container transport rate $D$ (provided containers are connected). When $D \sim \lambda$ the reaction dynamics in panels (b) and (c) should exhibit some similarities. For $D \gg \lambda$ one expects the same for the structures (a) and (c).

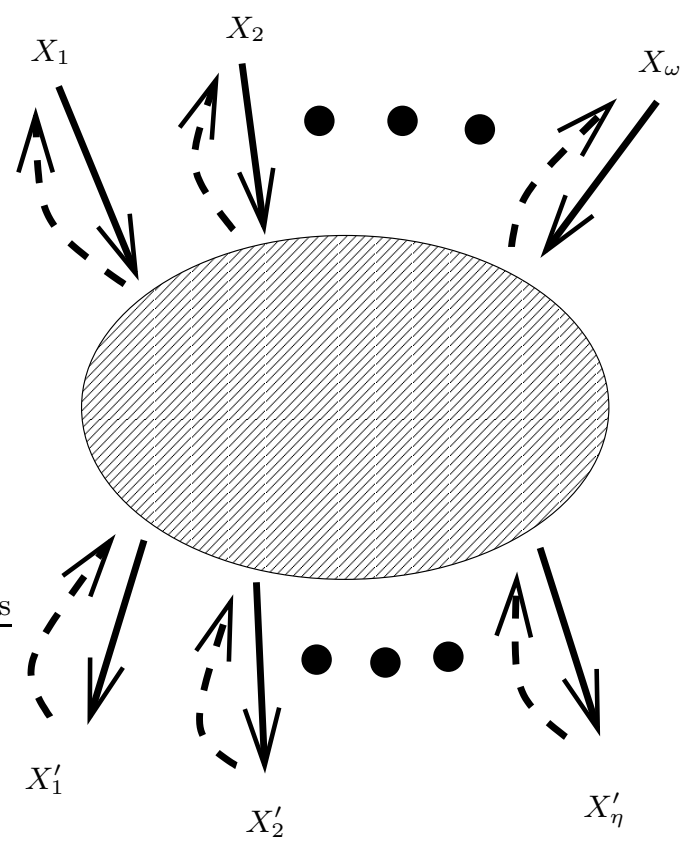

FIG. 2: The most general form of a reaction graph considered. Species $X_{1}, X_{2}, \ldots, X_{\omega}$ describe an inject pattern, a set of the molecules that are inserted into the system at $t=0$. This set is arranged into the vector $\iota$. Molecules denoted by $X_{1}^{\prime}, X_{2}^{\prime}, \ldots, X_{\eta}^{\prime}$ are the ones that have to be synthesized and are arranged into the vector $\boldsymbol{\pi}$. These are referred to as a task pattern. The shaded area in the middle denotes intermediate reaction steps. Dashed lines with arrows are allowed and result in appearance of loops in the reaction scheme. The speed of reaction is calculated by tracking instances when particles in the set $\boldsymbol{\pi}$ appear for the first time. The corresponding times $\mathcal{T}_{1}, \mathcal{T}_{2}, \ldots, \mathcal{T}_{\eta}$ are arranged into the vector $\mathcal{T}$. See section $\amalg$ for more details. 


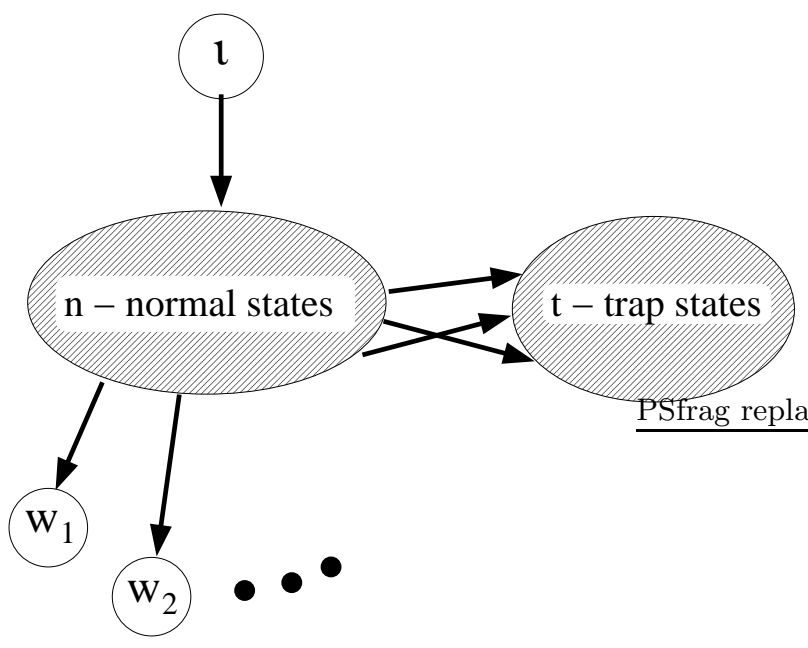

FIG. 3: The structure of the configuration space. Initially the system is in the state $\iota$. Three set of states are distinguished, a set of normal states $S_{n}$, a set of trap states $S_{t}$, and a set of window states $S_{w}$. See text for discussion.
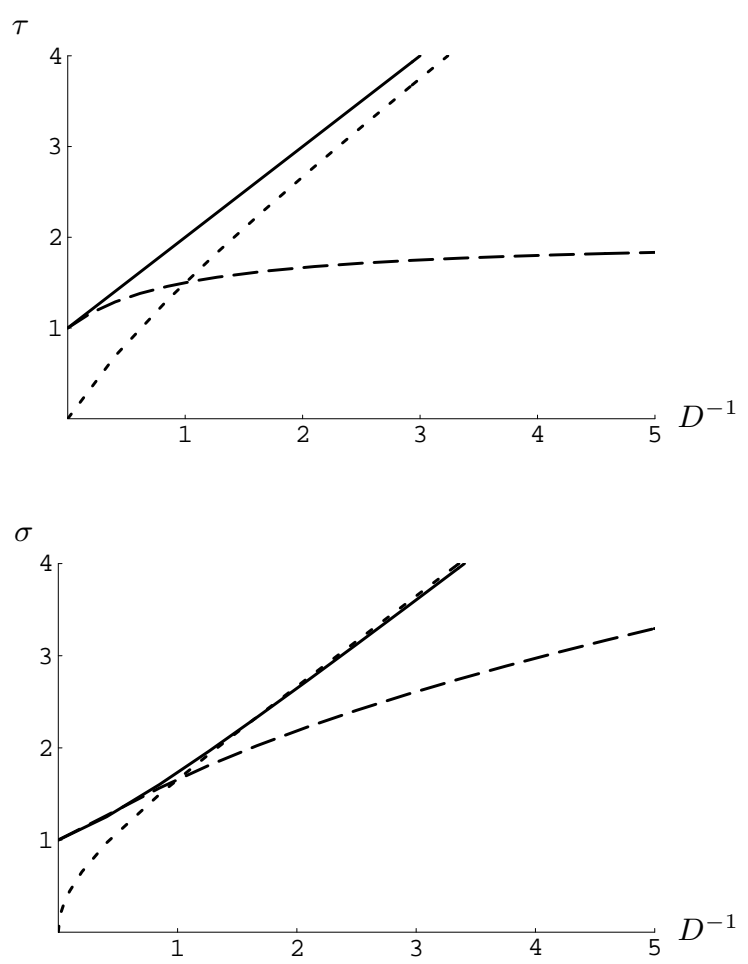

FIG. 4: The dependence of $\tau$ and $\sigma$ on $D^{-1} \sim \sqrt{l}$ for three organisms defined in table $(l$ is the length of a tube connecting containers). Curves describe $o_{1}$ (dotted line), $o_{2}$ (dashed) and $o_{3}$ (solid). See table $\square$ and section $\square$ for more details.

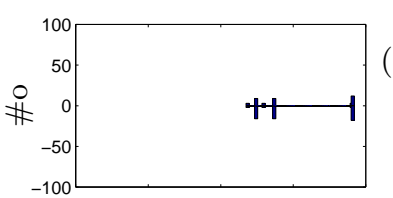

(a)
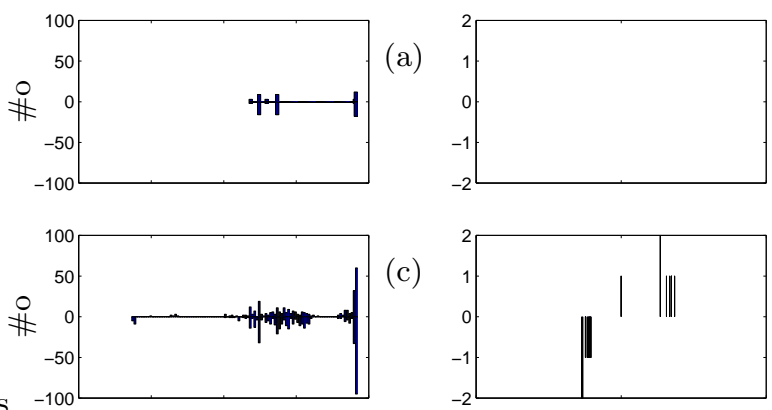

(c)

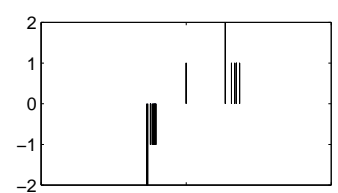

(d)

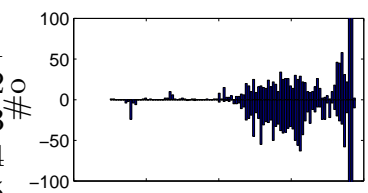

(e)

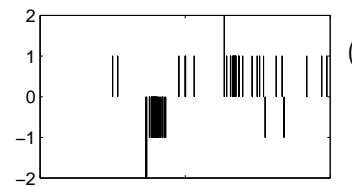

(f)

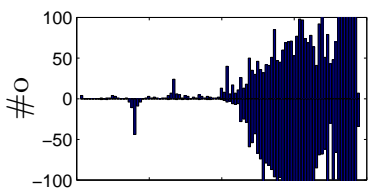

$(\mathrm{g})$

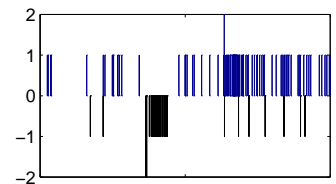

(h)

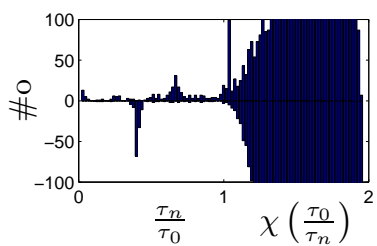

(i)

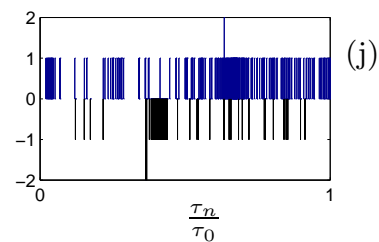

FIG. 5: Classification of organisms in ROGE ensemble. $\nu$ was calculated with $D_{n}=1 s^{-1}, D_{0}=3125 s^{-1}, \lambda=1 s^{-1}$, and $\xi=100$. Panels (a), (c), (e), (g) and (i) are histograms that depicts groups with similar reaction speed $\nu$. There are 100 classes of width 0.01 for $\nu$ from 1 to 2 . For $\nu>1$, instead of $\nu$, the value obtained from $\chi(\nu)=2-\ln 2 / \ln (1+\nu)$ is used. The function $\chi$ monotonically grows with $\nu$ and maps infinite interval $[1, \infty)$ onto the finite one $[1,2)$. In addition, this particular form for $\chi$ reveals more details in the region near $\nu=1$. Panels (b), (d), (f), (h), and (j) are discrete spectra (no histogram) for region $\nu \in[0,1]$. Panels in the same row have same value for the total number of particles in the system $N_{p}^{*}$ : (a) and (b) $N_{p}^{*}=1$, (c) and (d) $N_{p}^{*}=2$, (e) and (f) $N_{p}^{*}=3,(\mathrm{~g})$ and $(\mathrm{h}) N_{p}^{*}=4$, (i) and (j) $N_{p}^{*}=$ 5 . Negative value for number of organisms $(\# o)$ indicates that organism with particular value of $\nu$ contains at least one reaction that can be influenced through mechanism of suppression. Presence of suppressor lowers the reaction rate from $\lambda$ to $\lambda / \xi$ (see section $\amalg$ for details).

\section{Tables}


TABLE I: Analysis of a three cases where the total number of particles equals one and the number of containers equals two. These are simplest cases that one can consider with regard to the choices of $\boldsymbol{\iota}$ and $\boldsymbol{\pi}$. A reaction scheme used is $A \rightarrow B$ and $B \rightarrow A$, both with rate $\lambda$ (effects of catalyst or suppressor are absent since there is only one particle in the system at a time). The jump rate between containers is given by $D$. In the definition of $\iota$ and $\pi$ the content of containers is symbolically indicated as [content of $\left.C_{1}\right]$-[content of $\left.C_{2}\right]$.

\begin{tabular}{c|cc|cc}
\hline \hline org. & $\boldsymbol{\iota}$ & $\boldsymbol{\pi}$ & $\tau$ & $\sigma^{2}$ \\
\hline$o_{1}$ & {$[\mathrm{~A}]-[]$} & {[]$-[\mathrm{A}]$} & $\frac{2 D+\lambda}{D(D+\lambda)}$ & $\frac{2 D^{3}+5 D^{2} \lambda+3 D \lambda^{2}+\lambda^{3}}{D^{2} \lambda(D+\lambda)^{2}}$ \\
$o_{2}$ & {$[\mathrm{~A}]-[]$} & {$[\mathrm{B}]-[]$} & $\frac{D+2 \lambda}{\lambda(D+\lambda)}$ & $\frac{D^{3}+3 D^{2} \lambda+5 D \lambda^{2}+2 \lambda^{3}}{D \lambda^{2}(D+\lambda)^{2}}$ \\
$o_{3}$ & {$[\mathrm{~A}]-[]$} & {[]$-[\mathrm{B}]$} & $\frac{1}{D}+\frac{1}{\lambda}$ & $\frac{1}{D^{2}}+\frac{1}{D \lambda}+\frac{1}{\lambda^{2}}$ \\
\hline \hline
\end{tabular}

TABLE II: List of the best performers in the ROGE ensemble (first row). Couple of second best performers are also shown (second row). Last row contains organisms that are obtained by slightly perturbing winners from the first row.

\begin{tabular}{|c|c|c|c|}
\hline$N_{p}^{*}=2$ & $N_{p}^{*}=3$ & $N_{p}^{*}=4$ & $N_{p}^{*}=5$ \\
\hline$\left(o_{i, 2}\right)$ & $\left(o_{i, 3}\right)$ & $\left(o_{i, 4}\right)$ & $\left(o_{i, 5}\right)$ \\
\hline$A \stackrel{-A}{\longrightarrow} B, B \stackrel{+A}{\longrightarrow} A\left(R_{1}\right)$ & $A \stackrel{+A}{\longrightarrow} B\left(R_{3}\right)$ & $A \stackrel{+A}{\longrightarrow} B\left(R_{3}\right)$ & $A \stackrel{+A}{\longrightarrow} B\left(R_{3}\right)$ \\
\hline $\boldsymbol{\iota}=[\mathrm{A}]-[\mathrm{B}]$ & $\boldsymbol{\iota}=[\mathrm{A}]-[2 \mathrm{~A}]$ & $\boldsymbol{\iota}=[\mathrm{A}]-[3 \mathrm{~A}]$ & $\iota=[2 \mathrm{~A}]-[3 \mathrm{~A}]$ \\
\hline $\boldsymbol{\pi}=[2 \mathrm{~B}]-[\mathrm{]}$ or $[\mathrm{]}]-[2 \mathrm{~B}]$ & $\boldsymbol{\pi}=[2 \mathrm{~A}]-[\mathrm{B}]$ & $\boldsymbol{\pi}=[3 \mathrm{~A}]-[\mathrm{B}]$ & $\boldsymbol{\pi}=[4 \mathrm{~A}]-[\mathrm{B}]$ \\
\hline $\boldsymbol{\tau}_{n}=[18.55]-[]$ & $\boldsymbol{\tau}_{n}=[0.242]-[0.0355]$ & $\boldsymbol{\tau}_{n}=[0.021]-[0.01]$ & $\boldsymbol{\tau}_{n}=[0.014]-[0.00063]$ \\
\hline $\boldsymbol{\tau}_{0}=[50.91]-[]$ & $\tau_{0}=[0.000219]-[0.979]$ & $\boldsymbol{\tau}_{0}=[0.00045]-[0.95]$ & $\boldsymbol{\tau}_{0}=[0.010]-[0.91]$ \\
\hline$\nu=0.364$ & $\nu=0.250$ & $\nu=0.0248$ & $\nu=0.0192$ \\
\hline $\begin{array}{c}\left(o_{i i, 2}\right) \\
A \stackrel{-B}{\longrightarrow} B, B \stackrel{+A}{\longrightarrow} A\left(R_{2}\right) \\
\iota=[\mathrm{A}]-[\mathrm{B}], \boldsymbol{\pi}=[2 \mathrm{~B}]-[] \\
\text { or }[]-[2 \mathrm{~B}], \nu=0.368 \\
R_{1}, \iota=[]-[2 \mathrm{~A}], \\
\boldsymbol{\pi}=[2 \mathrm{~B}]-[], \nu=0.386 \\
R_{3}, \iota=[]-[2 \mathrm{~A}], \\
\boldsymbol{\pi}=[\mathrm{A}]-[\mathrm{B}], \nu=0.500\end{array}$ & $\begin{array}{c}\left(o_{i i, 3}\right) \\
R_{3}, \iota=[]-[3 \mathrm{~A}] \\
\boldsymbol{\pi}=[2 \mathrm{~A}]-[\mathrm{B}], \nu=0.268 ; \\
R_{1}, \iota=[2 \mathrm{~A}]-[\mathrm{B}] \\
\boldsymbol{\pi}=[\mathrm{A}]-[2 \mathrm{~B}] \\
R_{2}, \boldsymbol{\iota}=[2 \mathrm{~A}]-[\mathrm{B}] \\
\boldsymbol{\pi}=[2 \mathrm{~B}]-[\mathrm{A}] \text { or }[]-[\mathrm{A}, 2 \mathrm{~B}] \\
\nu \approx 0.386\end{array}$ & $\begin{array}{c}\left(o_{i i, 4}\right) \\
R_{3}, \iota=[2 \mathrm{~A}]-[2 \mathrm{~A}] \\
\boldsymbol{\pi}=[3 \mathrm{~A}]-[\mathrm{B}], \nu=0.0279 \\
R_{3}, \boldsymbol{\iota}=[]-[4 \mathrm{~A}] \\
\boldsymbol{\pi}=[3 \mathrm{~A}]-[\mathrm{B}], \nu=0.0358\end{array}$ & $\begin{array}{c}\left(o_{i i, 5}\right) \\
R_{3}, \iota=[2 \mathrm{~A}]-[3 \mathrm{~A}] \\
\boldsymbol{\pi}=[4 \mathrm{~A}, \mathrm{~B}]-[], \nu=0.0219 ; \\
R_{3}, \iota=[\mathrm{A}]-[4 \mathrm{~A}] \\
\boldsymbol{\pi}=[4 \mathrm{~A}]-[\mathrm{B}], \nu=0.0305\end{array}$ \\
\hline $\begin{array}{c}\left(o_{*, 2}\right) \\
R_{1} \text { or } R_{3}, \iota=[]-[2 \mathrm{~A}] \\
\boldsymbol{\pi}=[2 \mathrm{~A}]-[], \nu=3249 \text { or } 418\end{array}$ & $\begin{array}{c}\left(o_{*, 3}\right) \\
R_{3}, \iota=[\mathrm{A}]-[2 \mathrm{~A}] \\
\pi=[3 \mathrm{~A}]-[\quad], \nu=21.8\end{array}$ & $\begin{array}{c}\left(o_{*, 4}\right) \\
R_{3}, \iota=[\mathrm{A}]-[3 \mathrm{~A}] \\
\pi=[4 \mathrm{~A}]-[\quad], \nu=17.5\end{array}$ & $\begin{array}{c}\left(o_{*, 5}\right) \\
R_{3}, \iota=[2 \mathrm{~A}]-[3 \mathrm{~A}] \\
\pi=[5 \mathrm{~A}]-[\quad], \nu=10.4\end{array}$ \\
\hline
\end{tabular}

